\title{
Multidrug Efflux Pumps at the Crossroad between Antibiotic Resistance and Bacterial Virulence
}

\author{
Manuel Alcalde-Rico, Sara Hernando-Amado, Paula Blanco and José L. Martínez*
}

Departamento de Biotecnología Microbiana, Centro Nacional de Biotecnología, Consejo Superior de Investigaciones Científicas, Madrid, Spain

Multidrug efflux pumps can be involved in bacterial resistance to antibiotics at different levels. Some efflux pumps are constitutively expressed at low levels and contribute to intrinsic resistance. In addition, their overexpression may allow higher levels of resistance. This overexpression can be transient, in the presence of an effector (phenotypic resistance), or constitutive when mutants in the regulatory elements of the expression of efflux pumps are selected (acquired resistance). Efflux pumps are present in all cells, from human to bacteria and are highly conserved, which indicates that they are ancient elements in the evolution of different organisms. Consequently, it has been suggested that, besides antibiotic resistance, bacterial multidrug efflux pumps would likely contribute to other relevant processes of the microbial physiology. In the current article, we discuss some specific examples of the role that efflux pumps may have in the bacterial virulence of animals' and plants' pathogens, including the processes of intercellular communication. Based in these evidences, we propose that efflux pumps are at the crossroad between resistance and virulence of bacterial pathogens. Consequently, the comprehensive study of multidrug efflux pumps requires addressing these functions, which are of relevance for the bacterial-host interactions during infection.

Keywords: multidrug efflux pumps, quorum sensing, antibiotic resistance mechanisms, virulence, global regulation

\section{INTRODUCTION}

Multidrug resistance (MDR) efflux pumps are relevant elements belonging to the microbial repertoire that bacteria harbor for resisting the action of antimicrobial drugs (Piddock, 2006a; Vila and Martínez, 2008; Li et al., 2015; Jang, 2016). Indeed, several works have shown that these elements are involved in resistance of in vitro selected mutants as well as in the reduced susceptibility to antimicrobials of clinical isolates of different bacterial pathogens. The expression of efflux pumps is usually down regulated; only some of them present a substantial level of expression under regular growing conditions in the laboratory (Grkovic et al., 2001, 2002). However, constitutive high-level expression of these elements can be achieved by means of mutations in the elements regulating their expression. Transient high-level expression of efflux pumps can also be triggered in the presence of their effectors or under some specific growing conditions. In agreement with this situation, efflux pumps contribute to antibiotic resistance at three different levels: they can be involved in intrinsic resistance when presenting a basal level of expression under any condition. 
They can contribute to acquired resistance when mutants achieving high-level of expression of the efflux pumps are selected. Finally, they can contribute to transient, noninheritable, phenotypic resistance when bacteria are growing in the presence of an effector of the efflux pump or under growing conditions that trigger their overexpression. As reviewed in Hernando-Amado et al. (2016), efflux pumps are grouped in five structural families, namely the resistancenodulation-division (RND), the small multidrug resistance (SMR), the multi antimicrobial extrusion (MATE), the major facilitator superfamily (MFS), and the ATP-binding cassette (ABC) superfamilies. Whereas some efflux pumps can work independently of any other protein, mainly in the case of Grampositive organisms, in the case of Gram-negative organisms, they form tripartite complexes capable to traverse both bacterial membranes. These complexes include the inner-membrane efflux pump, a membrane fusion protein and an outer membrane protein.

When compared with other classical resistance genes, MDR efflux pumps present some specific features that support they should have other roles in the bacterial physiology besides their well-known involvement in antibiotic resistance. First, MDR efflux pumps are ubiquitous; they are present in all living cells, from humans to bacteria (Alonso et al., 1999; Alonso and Martinez, 2001; Gould et al., 2004; Sanchez et al., 2004). Second, the genes encoding them belong to the bacterial core genome in the sense that all (or most) members of a given species harbor the same efflux pumps (Alonso et al., 1999). Third, they are redundant; a single bacterial cell usually contains more than 10 different efflux pumps (Crossman et al., 2008). Fourth, they are rather unspecific; each efflux pump is able to extrude a variety of different substrates, including synthetic antibiotics as quinolones (Hernandez et al., 2011; Redgrave et al., 2014). Fifth, as above mentioned the expression of efflux pumps is tightly regulated; this regulation includes local regulators usually encoded upstream the structural genes of the operon encoding the efflux pump, as well as global regulators (Randall and Woodward, 2002; Luong et al., 2003; Nikaido et al., 2008; De Majumdar et al., 2013), frequently controlling the expression of a set of genes involved in the adaptation to a given ecosystem, as is the infected host. Sixth, at least in occasions, antibiotics are not good effectors of the expression of efflux pumps, whereas host-produced compounds as bile salts or plant-produced signals may induce the expression of MDR pumps (Rosenberg et al., 2003; Prouty et al., 2004; García-León et al., 2014). Altogether, these characteristics support that MDR efflux pumps are ancient elements (present in all organisms), important for the bacterial physiology (all members of a given species present the same, conserved efflux pumps), likely displaying different functions besides antibiotic resistance (a single microorganism contains a large number of different efflux pumps, with overlapping substrate ranges, including synthetic antibiotics not present in nature) and frequently integrated in complex response networks (they form part of global regulons and their expression is triggered by host produced compounds). In the present article we discuss some examples of the potential functions, besides antibiotic resistance, of MDR efflux pumps with a particular focus on the role that they may have in bacterial-host interactions in animals (humans) and plants as well as in intercellular signaling (Piddock, 2006b; Martinez et al., 2009; Alvarez-Ortega et al., 2013).

\section{EFFLUX PUMPS AND CELL-TO-CELL COMMUNICATION}

The capability to sense the environment and the organisms that are living in the same niche is critical to allow the microorganisms for choosing the best strategy to survive and colonize such niche. Along evolution, a battery of different mechanisms to sense the continuously changing environment has been selected in different microbial species. One of these mechanisms consists on the cell-to-cell communication systems. These inter-cellular signaling systems are based on the production of one or more low-molecular weight compounds which are sensed by molecular receptors of other cells, promoting a specific response in the target organism. In the bacterial world, this phenomenon is known as quorum sensing (QS) because it was initially described as a mechanism to sense the density of the bacterial population belonging to the same species present in a given habitat. The QS system allows the establishment of a cooperative genetic program of the whole population that increases the microbial efficacy for colonizing a given environment, including the infected host (Williams et al., 2007). The QS signal molecules (QSSMs) are constitutively produced at low quantities by all cells in the population. Their release outside the cells allows a progressive accumulation of QS signals in the intercellular space. The consequence is an increasingly production and accumulation of QSSMs while the population size increases, activating the QS response when the concentration of the signal reaches a threshold level. Further, these molecules (known as autoinducers) are able to induce their own production when they bind to their cognate transcriptional regulators, which produce a feedforward regulation circuit. The regulator-QSSM complex is the main responsible of triggering the QS response by increasing the expression of a large number of genes, including those encoding the autoinducer synthase enzymes, which further increases the QSSM production. This response coordinates a number of physiological changes at a population-scale level, which allows among other issues improving bacterial competition for nutrients with other species, forming morphological-resistance structures to overcome environmental threats or triggering the expression of virulence factors (Swift and Downie, 2001; Williams, 2007). Further, it has been shown that several QSSMs can be sensed by other species promoting responses mediated by inter-species communication processes (Williams et al., 2007; Shimada et al., 2013; Lee and Zhang, 2015), including eukaryotic cells (interkingdom signaling; Martinez, 2014). As above stated these QSSMs have to cross the cells membranes for their extracellular accumulation, and it has been reported that some MDR efflux pumps might be involved in their transport outside the cell. In this section, we will discuss the role of MDR efflux pumps in the modulation of intercellular signaling and how the acquisition of antibiotic resistance in mutants overexpressing efflux pumps 

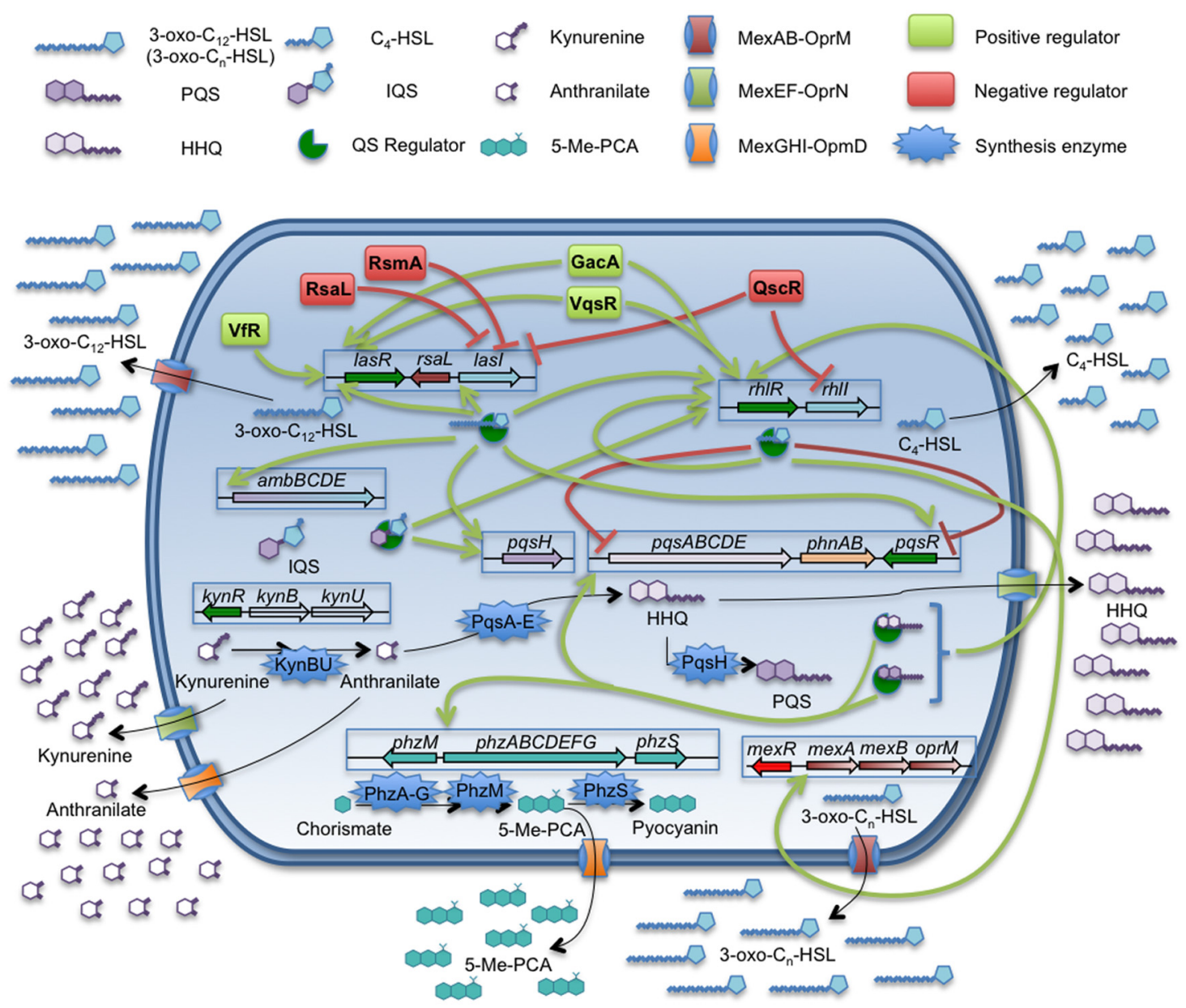

FIGURE 1 | Quorum sensing network and related efflux pumps in $\boldsymbol{P}$. aeruginosa. The QS signals produced by $P$. aeruginosa are: $3-\mathrm{O}$ - $\mathrm{xo}-\mathrm{C}_{12}-\mathrm{HSL}, \mathrm{C}_{4}-\mathrm{HSL}$, $\mathrm{PQS} / \mathrm{HHQ}$, and the recently discovered IQS. The figure sums up the complexity of the QS regulation network and the implications of MexAB-OprM, MexEF-OprN, and MexGHI-OpmD efflux pumps on the extrusion of QSSMs, their precursors or molecules which expression is QS-regulated. Despite of $\mathrm{C}_{4}-\mathrm{HSL}$ is able to cross the cell envelopes by free diffusion, the expression of mexAB-oprM is triggered in presence of this autoinducer. Further, this efflux pump is able to extrude 3-oxo- $\mathrm{C}_{12}-\mathrm{HSL}$ and others related 3-oxo- $\mathrm{C}_{n}-\mathrm{HSL}$. The MexEF-OprN system is able to extrude $\mathrm{HHQ}$ and kynurenine, a precursor of 4-alkyl-quinolones, having an impact on the QS response. In the case of MexGHI-OpmD, its role has been recently linked to the extrusion of 5-Me-PCA, a precursor of the phenazine pyocyanin, whose production is induced upon the QS response. However, it has been proposed that this system is able to efflux anthranilic acid, another AQs intermediate, which is toxic for the cell at high concentrations. P. aeruginosa is a good example for the potential role of efflux systems in modulating the cell-to-cell communication networks.

may challenge bacterial virulence through alterations in the diffusion of QS signals.

Pseudomonas aeruginosa is one of the most important opportunistic pathogens causing infections at hospitals as well as in cystic fibrosis patients (Buhl et al., 2015; Kaye and Pogue, 2015; Oliver et al., 2015; Talwalkar and Murray, 2016). This pathogen is able to produce different virulence factors, many of them being regulated by a hierarchically organized QS signaling system (Figure 1), which consists in three different and interconnected regulatory networks each one respectively governed by the transcriptional regulators LasR, RhlR, and PqsR (Lee and Zhang, 2015). These three QS regulators recognize respectively one of different QSSMs produced by $P$. aeruginosa: $N$-(3-oxododecanoyl)-L-homoserine lactone (3oxo-C12-HSL), N-butanoyl-L-homoserine lactone (C4-HSL), and Pseudomonas quinolone signal (PQS). The presence of an additional regulatory QS network has been recently described (Lee et al., 2013; Lee and Zhang, 2015). Despite being tightly regulated by the las system in standard conditions of growth, this new system is able to trigger the PQS and C4-HSL production in absence of the las system or under phosphate stress conditions. However, the mechanisms responsible of the regulation mediated by this QS system are not fully understood and consequently will not be discussed along the present review.

Some works have shown that the expression of different efflux pumps encoded in the $P$. aeruginosa chromosome may have an impact in the QS-regulation networks of this microorganism. For instance, the RND efflux pump MexAB-OprM is highly integrated within the las and $r h l$ QS regulons, since it has 
been shown that its expression can be induced by C4-HSL (Maseda et al., 2004; Sawada et al., 2004). In addition, this efflux pump is able to extrude the 3-oxo-C12-HSL QS signal (Evans et al., 1998; Pearson et al., 1999; Minagawa et al., 2012). In such a way, the constitutive high-level expression of MexABOprM entails the extrusion of 3-oxo-C12-HSL, increasing the concentration of this QS signal around the cell, but reducing its intracellular accumulation. Indeed, antibiotic resistant mutants overexpressing MexAB-OprM present defects in the production of several virulence factors and are impaired in their QS response (Evans et al., 1998).

It is important to highlight that the 3-oxo-C12-HSL autoinducer is not just an intra-specific signal compound but may also entail inter-kingdom signaling. For instance, it has been reported that this QS signal may inhibit the filamentous differentiation, which is linked to a virulent state of Candida albicans, a fungal pathogen commonly found in patients with P. aeruginosa infections (Hogan et al., 2004). Conversely, it has been also shown that the C. albicans QS compound farnesol in turn can inhibit the production of QSSMs and the production of virulence factors by $P$. aeruginosa (Méar et al., 2013), evidencing the relevance of these cell-to-cell communication "weapons" in the establishment of a competitive interaction between these two opportunistic pathogens. In addition to its role in inter-microbial interactions, a role as activator of the human immune system has been attributed to 3-oxo-C12-HSL. This autoinducer signal can act as a chemoattractant for polymorphonuclear neutrophils and is able to induce the expression of adhesion proteins and immunoglobulin receptors implicated in the recognition and localization of microbial infections (Smith et al., 2001; Zimmermann et al., 2006; Wagner et al., 2007). On the other hand, it has been shown that 3-oxo-C12-HSL can induce the apoptosis of neutrophils and macrophages (Tateda et al., 2003). The fact that 3-oxo-C12-HSL has been detected directly in the sputum of cystic fibrosis patients with $P$. aeruginosa infections indicates that this QS signal might be involved in in vivo interkingdom signaling (Erickson et al., 2002; Middleton et al., 2002).

Pseudomonas aeruginosa MDR strains overexpressing MexAB-OprM produce lower amounts of virulence factors as well as of 3-oxo-C12-HSL (Evans et al., 1998). In addition, mexAB-OprM defective mutants are avirulent in a mouse model of infection as well as in MDCK cells (Hirakata et al., 2002). These results support the notion that this efflux system is directly involved in the appropriate and coordinate las response needed for a successful host infection. Further, it has been proposed that this system is used by $P$. aeruginosa to optimize indirectly the specific binding of the LasR regulator to 3-oxo-C12-HSL by extruding other 3-oxo-Cn-HSL, which can affect the las network activation because they are able of competing with 3-oxo-C12HSL for the same binding site in LasR (Minagawa et al., 2012). All these intra- and interspecific responses mediated by 3-oxoC12-HSL suggest that MexAB-OprM is an important element modulating cell-to-cell communication and host-pathogen interactions. As a consequence, the acquisition of antimicrobial resistance through the overexpression of this system could have an impact, at several levels, on the virulence of $P$. aeruginosa.
Besides MexAB-OprM, other $P$. aeruginosa efflux pumps might be involved in the regulation of the expression of QSdependent virulence factors. One of them is MexEF-OprN, an efflux pump able to extrude both the QS signal HHQ (Lamarche and Deziel, 2011) and its precursor, kynurenine (Olivares et al., 2012), making it an important element in the QS response (Köhler et al., 2001). As we have mentioned above, the virulence and pathogenicity of $P$. aeruginosa is partially controlled by the PQS communication system. Therefore, it is not surprising that, as it happens in the case of MexAB-OprM, mutants overexpressing MexEF-OprN are affected in the production of QS-regulated virulence factors and, in consequence, they are impaired in the host infection process (Olivares et al., 2012). It is worth mentioning that the extrusion of kynurenine by this system could have an additional role in $P$. aeruginosa-host interaction. It has been shown that the expression of the MexEF-OprN system can be induced upon contact with human airway epithelial cells (Frisk et al., 2004). On the other hand, it has been suggested that $P$. aeruginosa production of kynurenine may have a role in the bacterial resistance to the toxic reactive oxygen species (ROS) produced by neutrophils, a key cell component in the innate immune system and in the inflammatory response in lung infections (Genestet et al., 2014). Based on this situation, it is possible that the increased expression of MexEF-OprN upon contact with the lung epithelial cells may allow $P$. aeruginosa to secrete high levels of kynurenine, thus promoting resistance against the neutrophil ROS production. This possibility, which has not been explored yet, would provide MexEF-OprN a new role in the infected lungs beyond the modulation of the QS response.

Another $P$. aeruginosa efflux pump with potential relevance in the virulence of this pathogen is MexGHI-OprD (Aendekerk et al., 2005; Dietrich et al., 2006). It has been shown that this efflux pump is able of extruding 5-methylphenazine-1-carboxylate (5-Me-PCA), a precursor of the phenazine pyocyanin, and anthranilate, the immediate precursor of PQS (Aendekerk et al., 2005). Further, the mexGHI-oprD expression could be induced by 5 -Me-PCA and, in consequence, is under the transcriptional control of the QS response. It is worth mentioning that the phenazine extruded by this efflux pump is required for biofilm development by P. aeruginosa (Sakhtah et al., 2016). If we take into consideration that biofilms are the regular way of growing of $P$. aeruginosa in the lungs of chronically infected patients (Martinez-Solano et al., 2008; Wagner and Iglewski, 2008), this indicates that MexGHI might have a relevant role in the adaptation of $P$. aeruginosa for colonizing that habitat.

Another relevant pathogen whose virulence has been associated to the extrusion of QS signals by efflux pumps is Burkholderia pseudomallei, the causal agent of melioidosis. Different works have suggested that the virulence of this pathogen is modulated by the stationary phase sigma factor RpoS in addition to the regulatory activity of a QS system based on both acyl-homoserine lactones (AHLs) and 2alkyl-4-quinolones (Ulrich et al., 2004; Wongtrakoongate et al., 2012; Butt et al., 2016). B. pseudomallei produces up to six different homoserine lactones: $\mathrm{N}$-octanoylhomoserine lactone (C8-HSL), $N$-decanoyl-homoserine lactone 
(C10-HSL), N-(3-hydroxy)-octanoyl-homoserine lactone (3$\mathrm{OH}$-C8-HSL), $\mathrm{N}$-(3-hydroxy)-decanoyl-homo-serine lactone (3-OH-C10-HSL), N-(3-oxo)-decanoyl-homoserine lactone (3-oxo-C10-HSL), and N-(3-oxo)-tetradecanoyl-homoserine lactone (3-oxo-C14-HSL). It has been reported that in the clinical strain KHW, the BpeAB-OprB efflux pump is strictly needed for the synthesis and full extrusion of these six AHLs and it has been suggested that another efflux pump, AmrAB-OprA could also be involved in the extrusion of 3-oxo-C10-HSL (Chan et al., 2007). In agreement with a potential role of these efflux pumps in QS communication, it has been shown that bpeAB-oprB expression is induced in presence of exogenous C8-HSL and C10-HSL (Chan and Chua, 2005). Moreover, a bpeAB-oprB defective mutant is affected in the expression of QS-dependent virulence factors as well as in biofilm formation. In addition, the mutant presents impaired invasiveness and cytotoxicity in both human macrophages and lung epithelial cells. Even though the role of BpeAB-OprB in virulence might be strain-dependent (Mima and Schweizer, 2010), all of the aforementioned results support the hypothesis that some efflux systems could be involved in the modulation of B. pseudomallei virulence and in the host-pathogen interactions through the extrusion of QS communication signals or by responding to their presence.

Other signaling networks involved in intra- and inter-specific communication are based in the use of indole and its derived compounds as signal molecules (Bommarius et al., 2013; Shimada et al., 2013; Lee J.H. et al., 2015). Indole is synthesized by plants and by many different bacteria and has different roles depending on the target species. Even though it was proposed that the efflux pump AcrEF could be implicated in indole export in Escherichia coli (Kawamura-Sato et al., 1999), other works have shown that indole is able to diffuse easily across the bacterial envelope (Gaede et al., 2005; Pinero-Fernandez et al., 2011) so that a clear role of efflux pumps in indole trafficking remains controversial. However, a crosstalk between virulence and efflux pumps-linked antibiotic resistance mediated through indole signaling is possible. Indeed, indole may affect the expression of efflux pumps, the resistance to antibiotics and the behavior of Salmonella enterica serovar Typhimurium when growing inside the host (Nikaido et al., 2012). This bacterial pathogen does not produce indole. However, it usually lives together with indole-producing species in the host and is able to respond to this signal (Nikaido et al., 2011). It is proposed that indole blocks the activity of the RamR transcriptional regulator through the interaction with its C-terminal domain, leading to the overexpression of RamA activator (Nikaido et al., 2011, 2012). This regulatory protein is able to induce the expression of $\operatorname{acr} A B$, $a c r E F$, and $t o l C$, in addition of repressing the expression of some virulence determinants (Bailey et al., 2010; Nikaido et al., 2012). This entails the development of a low-virulence phenotype, which presents, however, increased resistance to antibiotics as well as to host-produced toxic compounds as bile salts and fatty acids, a phenotype closer to a commensal behavior than to an infective one. This is another example of the relationship between the communication signals, with inter-specific function in this case and the expression of efflux pumps, a situation with clear implications in the virulence potential of bacterial pathogens.
Indeed, the role of AcrAB-TolC in the virulence of Salmonella goes beyond the acquired phenotype upon induction by indole, since, as described below, it has been shown that acr $A B$-tolC deficient mutants have reduced invasiveness in animal models (Buckley et al., 2006; Webber et al., 2009).

\section{MULTIDRUG EFFLUX PUMPS AND THEIR ROLE IN THE VIRULENCE OF HUMAN PATHOGENS}

During the course of an infection, a bacterial pathogen has to be capable of surviving from the anti-infective defense mechanisms of the host. These mechanisms include, among others, the production of a diverse set of antimicrobial compounds as fatty acids, peptides or even detergents as bile salts, which function is in food uptake, but also present antimicrobial activity (Fernando and Kumar, 2013). In addition of extruding antibiotics regularly used for treating bacterial infections, different MDR efflux pumps have the ability to extrude a wide variety of compounds, including those antimicrobials produced by the host as well as QS signals involved in the regulation of the expression of virulence determinants (see above). Consequently, these efflux pumps are relevant payers on both antibiotic resistance and virulence of bacterial populations (Piddock, 2006b; Martinez et al., 2009; Alvarez-Ortega et al., 2013).

In the case of enteric bacteria, it has been shown that the efflux of several host-derived antimicrobial compounds, such as bile salts, allows the colonization and promotes the bacterial adaptation to the animal intestinal tract. The best-studied system able to confer resistance to bile salts is the E. coli RND efflux pump AcrAB-TolC, which is also a major contributor to intrinsic resistance to antibiotics in this organism (Thanassi et al., 1997). Similar roles have been reported for different AcrAB homologs from other Enterobacteriaceae species, such as S. enterica serovar Typhimurium. In this case, it has been described that mutants lacking $a c r B$ and tolC are less proficient for adhering, invading and surviving in mouse monocyte macrophages. The same study reported that an $a c r B$ mutant was able to colonize chicks. However, it was unable to survive gastrointestinally, which suggests that AcrB is not particularly relevant for the early steps of the gut colonization but it is needed for gastrointestinal persistence. On the other hand, the tolC mutant was able to colonize and persist in the chicken intestinal tract, but with a much lower efficiency than the wild-type strain, which could be due to the bile hypersensitivity displayed by this mutant (Buckley et al., 2006). A more recent study, using total genome transcriptional analysis, showed that the inactivation of $\operatorname{acr} A$, $a c r B$, and tol $C$ rendered changes in the level of expression of several genes involved in bacterial pathogenicity, further supporting a crosstalk between resistance and virulence (Webber et al., 2009). For instance, the disruption of $a c r B$ or tolC led to a general repression of the SPI-1 pathogenicity island, which promotes the invasion of non-phagocytic intestinal epithelial cells, as well as bacterial survival and persistence within the host, while inactivation of $\operatorname{acr} A$ is associated with a repression of SPI-2, which promotes the survival and multiplication in 
phagocytic cells (Dieye et al., 2009). Therefore, inactivation of acr $B$ led to the inability to grow anaerobically, which would negatively impact in the capacity to survive in the host gut; as well as a reduced bacterial motility (Webber et al., 2009), which is also an important factor in the S. enterica serovar Typhimurium pathogenicity (Khoramian-Falsafi et al., 1990). As above stated, RND efflux systems are tripartite protein complexes formed by an inner membrane protein (the pump itself), an outer membrane protein and a linker, membrane fusion protein (Yamaguchi et al., 2015; Daury et al., 2016). The inner membrane and the membrane fusion proteins are always encoded in the same operon and are supposed to be specifically associated in each efflux pump, while the outer membrane protein can frequently form part of different efflux pumps. Consequently, while a differential response to the inactivation of the outer membrane protein of the complex (TolC in this case) as compared with the other members of the complex is conceivable, the observed differences between the $a c r A$ and $a c r B$ are more difficult to explain and suggests that a certain degree of trans-complementation between the components of different efflux pumps may happen. Although some information on this possibility has been published (Smith and Blair, 2014), this is a topic that remains to be studied in detail, although it merits to be addresses if we wish to understand in full the role that efflux pumps may play in the bacterial physiology besides antibiotic resistance.

An homologous of AcrAB is also present in the respiratory tract pathogen Moraxella catarrhalis. In addition of contributing to antibiotic resistance, this efflux pump is also involved in the efficient invasion of nasopharyngeal epithelial cells, since mutants lacking $\operatorname{acr} A, \operatorname{acr} B$, or tolC present decreased invasion levels as compared to the wild-type strain (Spaniol et al., 2015). This study also showed that exposure to cold shock $\left(26^{\circ} \mathrm{C}\right)$ led to an increase in the expression of the efflux pump genes (Spaniol et al., 2015). Since temperature is an important factor for the adaptation and survival in the respiratory tract, as well as for the colonization properties and the virulence of $M$. catarrhalis (Heiniger et al., 2005; Spaniol et al., 2013), these results indicate that the levels of expression of this efflux pump are controlled by cues with relevance for the infectious success of $M$. catarrhalis. This further supports that efflux pumps may form part of global regulatory networks that include resistance and virulence determinants among other elements (Randall and Woodward, 2002; Luong et al., 2003; Nikaido et al., 2008; De Majumdar et al., 2013).

The ability to persist and to replicate in bile-rich environments is also critical for the pathogenesis of the food-borne pathogen Listeria monocytogenes. Among the elements that contribute to its survival in bile salts are the MDR efflux pumps MdrM and MdrT, belonging to the MFS family (Quillin et al., 2011). Expression of both MDR efflux pumps is strongly induced by cholic acid, a bile component, but only the MdrT efflux pump is able to extrude this compound, which is toxic for mutants lacking this efflux pump. Besides, MdrT is an important virulence factor involved in the colonization of the gallbladder in vivo, since mutants lacking $m d r T$ are 100 -fold attenuated. This study also suggests that MdrM has a synergistic role with MdrT in L. monocytogenes liver colonization, although MdrM substrates have not been identified yet (Quillin et al., 2011).

RND efflux systems also play a role in the pathogenesis of Vibrio cholerae. It has been reported that VexAB, VexCD, VexIJK, and VexGH contribute not only to antimicrobial resistance, but also to the colonization of the infant mouse small intestine; deletion of these systems impair the colonization of the mice intestine by $V$. cholerae (Bina et al., 2008; Taylor et al., 2012). Besides being relevant factors for colonization, these efflux pumps are required for the expression of the genes that encode two of the most important virulence factors in $V$. cholerae: the cholera toxin (CT) and the toxin-coregulated pilus (TCP). In a mutant lacking $v e x B, v e x D$, vexH, and $v e x K$, the production of $\mathrm{CT}$ and TcpA (the pilin subunit of the TCP) is reduced by $45 \%$, while a six RND-null strain showed a 70\% reduction in the expression of these virulence determinants, suggesting that the remaining efflux pumps, VexF and VexM, also contribute to virulence in V. cholerae (Taylor et al., 2012).

Multidrug efflux systems are also relevant elements in the defense against oxidative stress produced in the host during phagocytosis. For example, the $\mathrm{ABC}$ family efflux pump $\mathrm{MacAB}$ is required for the survival of $S$. enterica serovar Typhimurium inside macrophages, where they are exposed to ROS. It has been observed that mutants lacking macAB showed an impaired intracellular replication in macrophages as compared with the wild-type parental strain and also failed in growing in the inflamed intestine, where neutrophils release ROS. Further, the same mutants were able to grow inside macrophages that do not produce ROS, which implies that MacAB is needed for S. enterica serovar Typhimurium replication inside macrophages and for the survival under oxidative stress conditions. Besides, the mac $A B$ deletion mutant had a defect in liver colonization in BALB/c mice after intraperitoneal infection, which indicates another function of this system in the infective program of $S$. enterica serovar Typhimurium (Bogomolnaya et al., 2013).

In addition to the role of efflux pumps in the interaction of bacterial pathogens with the compounds present in the host, they also may play a direct role in virulence. This is the case for the Mycobacterium tuberculosis RND proteins designated as MmpL (Mycobacterial membrane protein Large; Cole et al., 1998). The genome of M. tuberculosis encodes 13 of these proteins, which role seems to be transporting lipids for their incorporation on the cell envelope, providing protection against host-derived compounds and contributing to the bacterial virulence (Neyrolles and Guilhot, 2011). Domenech et al. (2005) examined the contribution of these proteins to the bacterium virulence by using a murine model of infection and mutants for each MmpL protein. Among these proteins, they found that MmpL4, MmpL7, MmpL8, and MmpL11 were required for the virulence maintenance, since there is an increase in the survival time when the host is infected with these mutants as compared with the wild-type strain. The attenuation of the mmpL7 mutant might be due to the lack of phthiocerol dimycocerosate (PDIM), which is an abundant wax of the outer cell matrix involved in the cell permeability (Camacho et al., 2001); and the mmpL8 mutant is deficient in SL-1 because MmpL8 is involved in the transport of SL-N, a precursor of 
SL-1 (Domenech et al., 2004). It has been shown that SL$\mathrm{N}$ stimulates human CD1b-restricted $\mathrm{T}$ cells (Gilleron et al., 2004), a feature that might explain the attenuation of the mmpL8 mutant if this molecule has a similar effect on murine CD1d-restricted T cells. The mechanisms by which mmpL4 and mmpL11 mutants were more attenuated have not been fully elucidated; however, it has been recently described a mutation in mmpL4a (Tyr842His) in Mycobacterium bolletii, which is responsible for the smooth-to-rough morphotype change, since MmpL4 is involved in the transport of glycopeptidolipid. This variant also contributes to the bacterial virulence in a zebrafish model (Bernut et al., 2016). The Tyr842 residue is conserved in all other mycobacterial MmpL4 orthologs and in all $13 \mathrm{MmpL}$ RND proteins in $M$. tuberculosis, which indicates the functional relevance of this residue (Szekely and Cole, 2016).

Biofilms are complex microbial associations attached to a variety of surfaces. Bacteria that grow forming biofilms are more resistant to antibiotics than planktonic cells, being also important elements in the bacterial virulence and pathogenesis. The link between antimicrobial tolerance on biofilms and efflux pumps has been reported in several microorganisms (Soto, 2013). For instance, in the opportunistic pathogen P. aeruginosa, the MerRlike regulator BrlR plays a role in the high-level tolerance to antimicrobials in biofilms because it is able to activate under these growing conditions the expression of the multidrug efflux pumps MexAB-OprM and MexEF-OprN (Liao et al., 2013). A novel efflux pump in $P$. aeruginosa involved in biofilm tolerance, named as PA1874-1877, has been identified. The expression of this efflux pump was 10 -fold increased in biofilms when compared with planktonic cells. Besides, deletion of the genes encoding this efflux pump resulted in an increased susceptibility to ciprofloxacin, gentamicin, and tobramycin (Zhang and Mah, 2008). Efflux pump expression can also impact the flagellar motility, which plays a relevant role in biofilm formation (Houry et al., 2012) and enhances pathogenicity by improving bacterial motility (Duan et al., 2013). In Stenotrophomonas maltophilia, another opportunistic pathogen, it has been observed that deletion of the RND efflux pump SmeYZ resulted in a reduced ability to form biofilm and the abolition of flagella formation. Besides, this deletion mutant was more susceptible to redox compounds, human serum and neutrophils, which indicates that this efflux pump is also involved in the protection against ROS (Lin et al., 2015).

Expression of efflux pumps not always enhance virulence; constitutive overexpression of these systems in antibiotic resistant mutants, can compromise the bacterial fitness and the virulence as well (Sanchez et al., 2002), indicating that the expression of these elements is finely regulated and deviations on this regulation, altering their expression below or above the physiological levels, may impair bacterial physiology and virulence. This is the case of the overexpression of the MDR efflux pumps MexCD-OprJ and MexEF-OprN in P. aeruginosa, which negatively affect the expression of the type III secretion system (T3SS; Linares et al., 2005). The T3SS is an important virulence mechanism, since bacteria are able to inject effector proteins manipulating the host cell function (Coburn et al., 2007). The effect of over-expression of efflux pumps on T3S was due to the lack of expression of exs $A$ gene, which is the transcriptional activator of the T3SS in $P$. aeruginosa (Linares et al., 2005). More recently, it was found that MexT, the positive regulator of MexEF, was able to repress the expression of the T3SS through the regulators MexS and PtrC (Jin et al., 2011), in such a way linking resistance and virulence within a single regulon.

Altogether, these works show that, in addition of being involved in antibiotic resistance, efflux pumps can participate in bacterial virulence as well. In some cases, a direct effect can be foreseen; this is the situation of efflux pumps able to extrude host-produced antimicrobial compounds. However, in other occasions the reasons behind the effect of efflux pumps on virulence are not so straightforward. In some cases, the effect on virulence is derived from the integration of the efflux pumps in a regulon that also includes virulence determinants. This could be the case of MexEF, a part of the MexT regulon that also includes the $P$. aeruginosa T3SS. Mutations in this global regulator will simultaneously alter antibiotic resistance and virulence, although the efflux pump itself is not directly involved in T3S. Other situation, also described for MexEF, is the capability of some efflux pumps for extruding intercellular signal molecules (see above) or their precursors (Olivares et al., 2012). If this efflux pump is abnormally expressed, the levels of expression of the genes belonging to the regulatory network (frequently including virulence genes) triggered by such signals will be also altered.

\section{THE FUNCTIONAL ROLE OF MULTIDRUG EFFLUX PUMPS IN PLANT-BACTERIA INTERACTIONS}

Multidrug efflux pumps, in addition of being relevant antibiotic resistance determinants, are relevant key players for the behavior of microorganisms in their natural (non-clinical) habitats. Indeed, whereas most studies in human pathogens have concentrated in the role on antibiotic resistance of these elements, the analyses of efflux pumps from plants pathogens or epiphytes, has mainly focused on their role in plant-bacteria or bacteriabacteria interactions. The rhizosphere is a natural ecosystem that includes a complex microbiome formed by microorganisms that live in contact with plants' roots. Roots' and other plants' exudates contain a large array of natural products, such as flavonoids, which confer them protection against microbial attack. However, different microorganisms have developed mechanisms to deal with the activity of those compounds. One of them is the flavonoid-responsive RND family of efflux pumps, which includes several members as MexAB-OprM from Pseudomonas syringae, AcrAB from Erwinia amylovora, AcrD from Erwinia chrysanthemi, IfeAB from Agrobacterium tumefaciens, XagID2689 from Xanthomonas axonopodis, SmeDEF from S. maltophilia, EmrAB from Sinorhizobium meliloti and BjG30 from Bradyrhizobium japonicum (Palumbo et al., 1998; Burse et al., 2004b; Vargas et al., 2011; Takeshima et al., 2013; García-León et al., 2014; Pletzer and Weingart, 2014; Rossbach et al., 2014; Chatnaparat et al., 2016). Some of these efflux pumps are implicated in plant colonization, whereas 
some others are involved in bacteria/plant symbiosis processes. As stated before MexAB-OprM is described as a significant determinant of multidrug resistance in $P$. aeruginosa (Poole, 2001) having a basal expression level enough to contribute to the intrinsic antimicrobial resistance of these bacteria ( $\mathrm{Li}$ et al., 1995) and it also has a role in virulence. Indeed, as above described, mutant strains overexpressing this efflux pump are less virulent because of the extrusion of the QS homoserine lactone 3-oxo-C 12 -HSL (Minagawa et al., 2012). In addition of extruding antibiotics or QS signaling molecules, MexAB-OprM is able to pump out plant antimicrobial compounds from leaves of Melaleuca alternifolia, supporting that, in addition of its role in clinical settings, this efflux pump (and several others, see below) may allow bacterial survival in a vegetal environment (Papadopoulos et al., 2008). Indeed, this efflux pump is required for the efficient colonization of tomato plants by $P$. syringae, since the inactivation MexAB-OprM lead to a defective colonization capacity of the plant by this bacterial species (Vargas et al., 2011). In line with the role of efflux pumps in a general bacterial response to the plant antimicrobial defense, it has been shown that flavonoids are inducers and substrates of this transporter (Vargas et al., 2011). In addition, the same flavonoids are able to inhibit the GacS/GacA two component system (TCS) of $P$. syringae, which is implicated, among other regulatory elements, in the activation of the motility and the T3SS in this species (Chatterjee et al., 2003). In fact, the absence of MexAB-OprM and the accumulation of flavonoids inside bacteria lead to a reduction of swarming and swimming motility and a significant impairment in the production of flagella and T3S (Vargas et al., 2013). Therefore, when the amount of plant flavonoids is enough to reduce bacterial virulence by inhibiting $\mathrm{GacS} / \mathrm{GacA}, \quad$. syringae may extrude such flavonoids using the MexAB efflux pump (which expression is now induced), shrewdly regulating the intracellular level of flavonoids and consequently, ensuring the viability of the bacteria by the de-repression of this TCS. This is an elegant example of the adaptive co-evolution of plant resistance and pathogen virulence in which the role of this efflux pump goes beyond being a mere detoxification system (Figure 2).

Another plant-pathogenic bacteria is E. amylovora, an Enterobacteria that causes fire blight on apple and pear trees. The flavonoid inducible AcrAB-TolC efflux pump from E. amylovora confers resistance, in addition to antibiotics, to plant compounds as phytoalexins and flavonoids (phloretin, naringenin, and quercetin) leading to the successful colonization of plants (Burse et al., 2004b; Maggiorani Valecillos et al., 2006; Al-Karablieh et al., 2009). Moreover, an AcrAB deletion mutant has a strong reduction of E. amylovora virulence in apple plants (Burse et al., 2004b). A close homolog of AcrB is AcrD, an efflux pump that is also induced by plant flavonoids, such as luteolin, but whose deletion mutant exhibits full virulence on apple and pear fruits (Pletzer and Weingart, 2014). E. chrysanthemi constitutes another example in which efflux pumps are required to infect plants. A tolC mutant of E. chrysanthemi is unable to extrude the plant antimicrobial compound berberine, being then unable to cause plant tissue maceration. Moreover, TolC plays a role also modulating the fitness of the bacteria growing in the microbial community (Barabote et al., 2003), indicating that efflux pumps might also be involved in inter-microbial interactions (see below). In addition of plant-derived antimicrobials, the AcrAB efflux pump of E. chrysanthemi is inducible by salicylic acid (SA), an important plant hormone implicated in local and systemic plant resistance (Ravirala et al., 2007). SA and others plant phenolic acids are able of reducing the expression of the T3SS, by inhibiting the GacS/A pathway, in P. syringae (Lee J.S. et al., 2015), suggesting that a role of efflux pumps in non-clinical ecosystems might be to adjust the intracellular level of plant signals, as a mechanism to deal with plant defense systems. Other efflux pump that has a role in plant colonization is IfeAB from A. tumefaciens, which extrudes coumestrol bestowing measurable ecological benefits to this bacterium in flavonoids rich environments. $X$. axonopodis pv. glycines is another plant pathogen, causative of bacterial pustule of Glycine max, one of the most important diseases in soybean. It contains another flavonoid inducible RND efflux pump, called XagID2689 (Chatnaparat et al., 2016), which in addition of being induced by flavonoids contributes as well to flavonoids' resistance; its deletion strongly reduces bacterial virulence in soybean. Moreover, this deletion mutant shows a higher susceptibility than the wild-type parental counterpart to the isoflavonoids phloretin, naringenin and berberine, as well as to the antibiotics acriflavine and tetracycline, suggesting a role in the intracellular reduction of the levels of several compounds produced by soybean and involved in its antimicrobial response program. In S. maltophilia, the SmeDEF efflux pump, which is the most important quinolone resistance determinant of this microorganism (Alonso and Martinez, 2000, 2001; Zhang et al., 2001; Garcia-Leon et al., 2014), is induced by flavonoids, that are able to bind to its SmeT repressor (García-León et al., 2014); in addition, a mutant lacking smeE is unable to colonize the roots of Arabidopsis plants (GarcíaLeón et al., 2014), further supporting that MDR efflux pumps, with a relevant role for antibiotic resistance at clinical settings, might have been selected in nature for different ecological purposes.

In addition of easening the bacterial plants' colonization and infection, efflux pumps have also a role in the interactions between plants and their symbiotic bacteria. For example, EmrAB from S. meliloti is an inducible flavonoid efflux pump with a role in symbiosis with Medicago sativa; the symbiotic process is impaired when the regulator of this efflux system, the TetR repressor EmrR, is deleted (Rossbach et al., 2014; Santos et al., 2014). Other examples of efflux pumps with a role in symbiotic nitrogen-fixation activity processes in G. max are $B d e A B$ and BjG30 from B. japonicum (Lindemann et al., 2010; Takeshima et al., 2013). BdeAB deficient mutants, in addition of presenting symbiotic defects, are more susceptible to aminoglycosides, highlighting the multifunctional role of efflux pumps (Lindemann et al., 2010).

Efflux pumps have also an important role in the intermicrobial interactions in the host plant and in its rhizosphere, where each bacteria have to compete for space and nutrients to survive. As with antibiotic resistance mechanisms, that can be considered as a colonization factor in the treated 


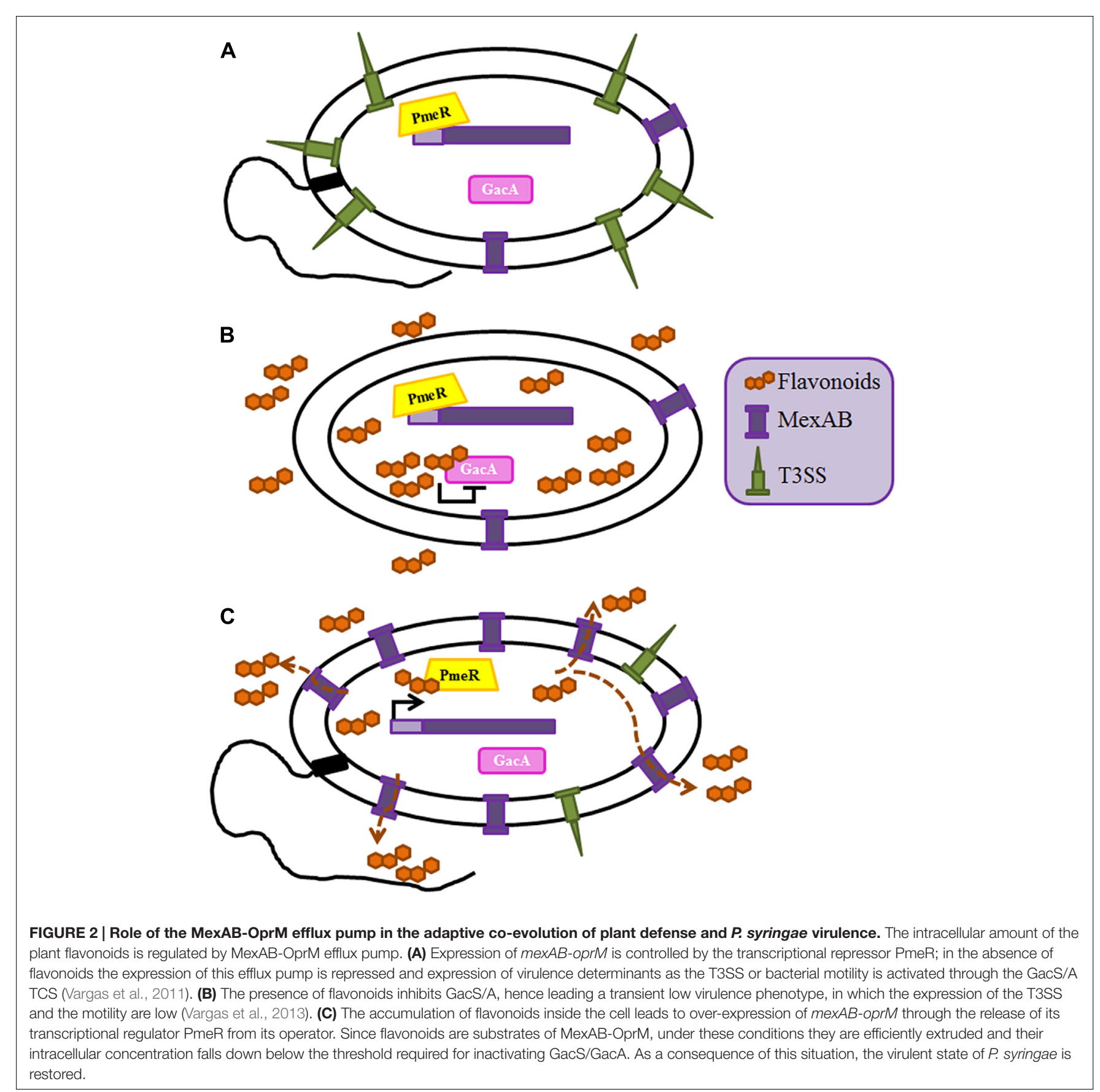

patient, where antibiotics are present (Martinez and Baquero, 2002), resistance to antimicrobials produced by epiphytes may be considered as a colonization tool of phytopathogenic bacteria and vice versa. If the epiphytes can inhibit the phytopathogens, the plant will be protected from infection, whereas if pathogens can inhibit epiphytes, this situation will provide a colonization advantage and hence better possibilities for infecting the plant. Resistance to compounds produced by bacterial competitors will then increase the chances for colonization of the epiphyte or the pathogen, with important consequence in terms of crops protection. This is the case of the efflux pump NorM from E. amylovora that, in contrast to the previously mentioned efflux pump AcrAB from E. amylovora (Burse et al., 2004b), is not able to extrude the high amount of isoflavonoids that produce the members of the Rosaceae family (Burse et al., 2004a). Moreover, a norM-deficient mutant causes comparable symptoms as the wild-type parental counterpart in plant tissues, indicating that this efflux pump does not contribute to virulence of $E$. amylovora against apple plants. Nevertheless, while the NorM efflux pump does not directly contribute to virulence E. amylovora, it has an important role extruding toxic molecules produced by Pantoea 
agglomerans (Pusey et al., 2011), an epiphytic bacteria that is an excellent colonizer of stigmas of apple and pear and it may effectively inhibit the multiplication of E. amylovora when both microorganisms co-colonize rosaceous (Burse et al., 2004a; Piddock, 2006b), hence constituting a potential biocontrol agent for fire blight (Kim et al., 2012). It is worth mentioning that NorM is involved in the capability E. amylovora to reach highdensity populations at low temperatures. Indeed, at $18^{\circ} \mathrm{C}$ the growth of an E. amylovora norM mutant is significantly impaired as compared with the wild-type strain. In addition, the level of expression of nor $M$ is twofold greater at $18^{\circ} \mathrm{C}$ gene than at $28^{\circ} \mathrm{C}$ (Burse et al., 2004a). Altogether these results suggest that the intrinsic resistance of E. amylovora to $P$. agglomerans competition at $18^{\circ} \mathrm{C}$ may be due to the increased expression level of norM, which might allow the colonization by E. amylovora of the stigma surface of the blossom when it coexists with $P$. agglomerans. These evidences lead to hypothesize that the utilization of $P$. agglomerans as a biocontrol mechanism for fire blight would be compromised at low temperatures or by mutations causing of overexpression of norM in E. amylovora.

\section{REFERENCES}

Aendekerk, S., Diggle, S. P., Song, Z., Hoiby, N., Cornelis, P., Williams, P., et al. (2005). The MexGHI-OpmD multidrug efflux pump controls growth, antibiotic susceptibility and virulence in Pseudomonas aeruginosa via 4-quinolonedependent cell-to-cell communication. Microbiology 151(Pt. 4), 1113-1125. doi: 10.1099/mic.0.27631-0

Al-Karablieh, N., Weingart, H., and Ullrich, M. S. (2009). The outer membrane protein TolC is required for phytoalexin resistance and virulence of the fire blight pathogen Erwinia amylovora. Microb. Biotechnol. 2, 465-475. doi: 10.1111/j.1751-7915.2009.00095.x

Alonso, A., and Martinez, J. L. (2000). Cloning and characterization of SmeDEF, a novel multidrug efflux pump from Stenotrophomonas maltophilia. Antimicrob. Agents Chemother. 44, 3079-3086. doi: 10.1128/AAC.44.11.30793086.2000

Alonso, A., and Martinez, J. L. (2001). Expression of multidrug efflux pump SmeDEF by clinical isolates of Stenotrophomonas maltophilia. Antimicrob. Agents Chemother. 45, 1879-1881. doi: 10.1128/AAC.45.6. 1879-1881.2001

Alonso, A., Rojo, F., and Martinez, J. L. (1999). Environmental and clinical isolates of Pseudomonas aeruginosa show pathogenic and biodegradative properties irrespective of their origin. Environ. Microbiol. 1, 421-430. doi: 10.1046/j.14622920.1999.00052.x

Alvarez-Ortega, C., Olivares, J., and Martínez, J. L. (2013). RND multidrug efflux pumps: what are they good for? Front. Microbiol. 4:7. doi: 10.3389/fmicb.2013.00007

Bailey, A. M., Ivens, A., Kingsley, R., Cottell, J. L., Wain, J., and Piddock, L. J. (2010). RamA, a member of the AraC/XylS family, influences both virulence and efflux in Salmonella enterica serovar typhimurium. J. Bacteriol. 192, 1607-1616. doi: 10.1128/JB.01517-09

Barabote, R. D., Johnson, O. L., Zetina, E., San Francisco, S. K., Fralick, J. A., and San Francisco, M. J. (2003). Erwinia chrysanthemi tolC is involved in resistance to antimicrobial plant chemicals and is essential for phytopathogenesis. J. Bacteriol. 185, 5772-5778. doi: 10.1128/JB.185.19.57725778.2003

Bernut, A., Viljoen, A., Dupont, C., Sapriel, G., Blaise, M., Bouchier, C., et al. (2016). Insights into the smooth-to-rough transitioning in Mycobacterium bolletii unravels a functional Tyr residue conserved in all mycobacterial MmpL family members. Mol. Microbiol. 99, 866-883. doi: 10.1111/mmi. 13283

Bina, X. R., Provenzano, D., Nguyen, N., and Bina, J. E. (2008). Vibrio cholerae RND family efflux systems are required for antimicrobial resistance, optimal

\section{AUTHOR CONTRIBUTIONS}

All authors listed, have made substantial, direct and intellectual contribution to the work, and approved it for publication.

\section{FUNDING}

Work in our laboratory is supported by grants from the Spanish Ministry of Economy and Competitiveness (BIO2014-54507$\mathrm{R}$ and JPI Water StARE JPIW2013-089-C02-01); from Madrid Autonomous Community [S2010/BMD2414 (PROMPT)]; and from the Instituto de Salud Carlos III [Spanish Network for Research on Infectious Diseases (REIPI RD12/0015)]. MA-R and $\mathrm{PB}$ are recipients of FPI fellowships from MINECO.

\section{ACKNOWLEDGMENT}

Thanks are given to Mary Higgins for grammar corrections.

virulence factor production, and colonization of the infant mouse small intestine. Infect. Immun. 76, 3595-3605. doi: 10.1128/IAI.01620-07

Bogomolnaya, L. M., Andrews, K. D., Talamantes, M., Maple, A., Ragoza, Y., Vazquez-Torres, A., et al. (2013). The ABC-type efflux pump MacAB protects Salmonella enterica serovar typhimurium from oxidative stress. MBio 4, e63013. doi: $10.1128 / \mathrm{mBio} .00630-13$

Bommarius, B., Anyanful, A., Izrayelit, Y., Bhatt, S., Cartwright, E., Wang, W., et al. (2013). A family of indoles regulate virulence and Shiga toxin production in pathogenic E. coli. PLOS ONE 8:e54456. doi: 10.1371/journal.pone. 0054456

Buckley, A. M., Webber, M. A., Cooles, S., Randall, L. P., La Ragione, R. M., Woodward, M. J., et al. (2006). The AcrAB-TolC efflux system of Salmonella enterica serovar typhimurium plays a role in pathogenesis. Cell Microbiol. 8, 847-856. doi: 10.1111/j.1462-5822.2005.00671.x

Buhl, M., Peter, S., and Willmann, M. (2015). Prevalence and risk factors associated with colonization and infection of extensively drug-resistant Pseudomonas aeruginosa: a systematic review. Expert Rev. Anti Infect. Ther. 13, 1159-1170. doi: 10.1586/14787210.2015.1064310

Burse, A., Weingart, H., and Ullrich, M. S. (2004a). NorM, an Erwinia amylovora multidrug efflux pump involved in in vitro competition with other epiphytic bacteria. Appl. Environ. Microbiol. 70, 693-703. doi: 10.1128/AEM.70.2.693703.2004

Burse, A., Weingart, H., and Ullrich, M. S. (2004b). The phytoalexin-inducible multidrug efflux pump AcrAB contributes to virulence in the fire blight pathogen, Erwinia amylovora. Mol. Plant Microbe Interact. 17, 43-54. doi: 10.1094/MPMI.2004.17.1.43

Butt, A., Halliday, N., Williams, P., Atkins, H. S., Bancroft, G. J., and Titball, R. W. (2016). Burkholderia pseudomallei kynB plays a role in AQ production, biofilm formation, bacterial swarming and persistence. Res. Microbiol. 167, 159-167. doi: 10.1016/j.resmic.2015.11.002

Camacho, L. R., Constant, P., Raynaud, C., Laneelle, M. A., Triccas, J. A., Gicquel, B., et al. (2001). Analysis of the phthiocerol dimycocerosate locus of Mycobacterium tuberculosis. Evidence that this lipid is involved in the cell wall permeability barrier. J. Biol. Chem. 276, 19845-19854. doi: 10.1074/jbc.M100662200

Chan, Y. Y., Bian, H. S., Tan, T. M., Mattmann, M. E., Geske, G. D., Igarashi, J., et al. (2007). Control of quorum sensing by a Burkholderia pseudomallei multidrug efflux pump. J. Bacteriol. 189, 4320-4324. doi: 10.1128/JB. 00003-07

Chan, Y. Y., and Chua, K. L. (2005). The Burkholderia pseudomallei BpeABOprB efflux pump: expression and impact on quorum sensing and virulence. J. Bacteriol. 187, 4707-4719. doi: 10.1128/JB.187.14.4707-4719.2005 
Chatnaparat, T., Prathuangwong, S., and Lindow, S. E. (2016). Global pattern of gene expression of Xanthomonas axonopodis pv. glycines within soybean leaves. Mol. Plant Microbe Interact. 29, 508-522. doi: 10.1094/mpmi-01-160007-r

Chatterjee, A., Cui, Y., Yang, H., Collmer, A., Alfano, J. R., and Chatterjee, A. K. (2003). GacA, the response regulator of a two-component system, acts as a master regulator in Pseudomonas syringae pv. tomato DC3000 by controlling regulatory RNA, transcriptional activators, and alternate sigma factors. Mol. Plant Microbe Interact. 16, 1106-1117. doi: 10.1094/mpmi.2003.16. 12.1106

Coburn, B., Sekirov, I., and Finlay, B. B. (2007). Type III secretion systems and disease. Clin. Microbiol. Rev. 20, 535-549. doi: 10.1128/CMR.00013-07

Cole, S. T., Brosch, R., Parkhill, J., Garnier, T., Churcher, C., Harris, D., et al. (1998). Deciphering the biology of Mycobacterium tuberculosis from the complete genome sequence. Nature 393, 537-544. doi: 10.1038/31159

Crossman, L. C., Gould, V. C., Dow, J. M., Vernikos, G. S., Okazaki, A., Sebaihia, M., et al. (2008). The complete genome, comparative and functional analysis of Stenotrophomonas maltophilia reveals an organism heavily shielded by drug resistance determinants. Genome Biol. 9:R74. doi: 10.1186/gb-2008-94-r74

Daury, L., Orange, F., Taveau, J. C., Verchere, A., Monlezun, L., Gounou, C., et al. (2016). Tripartite assembly of RND multidrug efflux pumps. Nat. Commun. 7:10731. doi: $10.1038 /$ ncomms 10731

De Majumdar, S., Veleba, M., Finn, S., Fanning, S., and Schneiders, T. (2013). Elucidating the regulon of multidrug resistance regulator RarA in Klebsiella pneumoniae. Antimicrob. Agents Chemother. 57, 1603-1609. doi: 10.1128/AAC.01998-12

Dietrich, L. E., Price-Whelan, A., Petersen, A., Whiteley, M., and Newman, D. K. (2006). The phenazine pyocyanin is a terminal signalling factor in the quorum sensing network of Pseudomonas aeruginosa. Mol. Microbiol. 61, 1308-1321. doi: 10.1111/j.1365-2958.2006.05306.x

Dieye, Y., Ameiss, K., Mellata, M., and Curtiss, R. III (2009). The Salmonella Pathogenicity Island (SPI) 1 contributes more than SPI2 to the colonization of the chicken by Salmonella enterica serovar typhimurium. BMC Microbiol. 9:3. doi: 10.1186/1471-2180-9-3

Domenech, P., Reed, M. B., and Barry, C. E. III (2005). Contribution of the Mycobacterium tuberculosis $\mathrm{MmpL}$ protein family to virulence and drug resistance. Infect. Immun. 73, 3492-3501. doi: 10.1128/IAI.73.6.34923501.2005

Domenech, P., Reed, M. B., Dowd, C. S., Manca, C., Kaplan, G., and Barry, C. E. III (2004). The role of MmpL8 in sulfatide biogenesis and virulence of Mycobacterium tuberculosis. J. Biol. Chem. 279, 21257-21265. doi: 10.1074/jbc.M400324200

Duan, Q., Zhou, M., Zhu, L., and Zhu, G. (2013). Flagella and bacterial pathogenicity. J. Basic Microbiol. 53, 1-8. doi: 10.1002/jobm. 201100335

Erickson, D. L., Endersby, R., Kirkham, A., Stuber, K., Vollman, D. D., Rabin, H. R., et al. (2002). Pseudomonas aeruginosa quorum-sensing systems may control virulence factor expression in the lungs of patients with cystic fibrosis. Infect. Immun. 70, 1783-1790. doi: 10.1128/IAI.70.4.1783-1790.2002

Evans, K., Passador, L., and Srikumar, R. (1998). Influence of the MexABOprM multidrug efflux system on quorum sensing in Pseudomonas aeruginosa. J. Bacteriol. 180, 5443-5447.

Fernando, D. M., and Kumar, A. (2013). Resistance-nodulation-division multidrug efflux pumps in gram-negative bacteria: role in virulence. Antibiotics (Basel) 2, 163-181. doi: 10.3390/antibiotics2010163

Frisk, A., Schurr, J. R., Wang, G., Bertucci, D. C., Marrero, L., Hwang, S. H., et al. (2004). Transcriptome analysis of Pseudomonas aeruginosa after interaction with human airway epithelial cells. Infect. Immun. 72, 5433-5438. doi: 10.1128/IAI.72.9.5433-5438.2004

Gaede, H. C., Yau, W. M., and Gawrisch, K. (2005). Electrostatic contributions to indole-lipid interactions. J. Phys. Chem. B 109, 13014-13023. doi: 10.1021/jp0511000

García-León, G., Hernández, A., Hernando-Amado, S., Alavi, P., Berg, G., and Martínez, J. L. (2014). A function of SmeDEF, the major quinolone resistance determinant of Stenotrophomonas maltophilia, is the colonization of plant roots. Appl. Environ. Microbiol. 80, 4559-4565. doi: 10.1128/AEM. 01058-14
Garcia-Leon, G., Salgado, F., Oliveros, J. C., Sanchez, M. B., and Martinez, J. L. (2014). Interplay between intrinsic and acquired resistance to quinolones in Stenotrophomonas maltophilia. Environ. Microbiol. 16, 1282-1296. doi: $10.1111 / 1462-2920.12408$

Genestet, C., Le Gouellec, A., Chaker, H., Polack, B., Guery, B., Toussaint, B., et al. (2014). Scavenging of reactive oxygen species by tryptophan metabolites helps Pseudomonas aeruginosa escape neutrophil killing. Free Radic. Biol. Med. 73, 400-410. doi: 10.1016/j.freeradbiomed.2014.06.003

Gilleron, M., Stenger, S., Mazorra, Z., Wittke, F., Mariotti, S., Bohmer, G., et al. (2004). Diacylated sulfoglycolipids are novel mycobacterial antigens stimulating CD1-restricted T cells during infection with Mycobacterium tuberculosis. J. Exp. Med. 199, 649-659. doi: 10.1084/jem.20031097

Gould, V. C., Okazaki, A., Howe, R. A., and Avison, M. B. (2004). Analysis of sequence variation among smeDEF multi drug efflux pump genes and flanking DNA from defined 16S rRNA subgroups of clinical Stenotrophomonas maltophilia isolates. J. Antimicrob. Chemother. 54, 348-353. doi: 10.1093/jac/dkh367

Grkovic, S., Brown, M. H., and Skurray, R. A. (2001). Transcriptional regulation of multidrug efflux pumps in bacteria. Semin. Cell Dev. Biol. 12, 225-237. doi: $10.1006 / \mathrm{scdb} .2000 .0248$

Grkovic, S., Brown, M. H., and Skurray, R. A. (2002). Regulation of bacterial drug export systems. Microbiol. Mol. Biol. Rev. 66, 671-701. doi: 10.1128/MMBR.66.4.671-701.2002

Heiniger, N., Troller, R., Meier, P. S., and Aebi, C. (2005). Cold shock response of the UspA1 outer membrane adhesin of Moraxella catarrhalis. Infect. Immun. 73, 8247-8255. doi: 10.1128/IAI.73.12.8247-8255.2005

Hernandez, A., Sanchez, M. B., and Martinez, J. L. (2011). Quinolone resistance: much more than predicted. Front. Microbiol. 2:22. doi: 10.3389/fmicb.2011.00022

Hernando-Amado, S., Blanco, P., Alcalde-Rico, M., Corona, F., Reales-Calderón, J. A., Sánchez, M. B., et al. (2016). Multidrug efflux pumps as main players in intrinsic and acquired resistance to antimicrobials. Drug Resist. Updat. 28, 13-27. doi: 10.1016/j.drup.2016.06.007

Hirakata, Y., Srikumar, R., Poole, K., Gotoh, N., Suematsu, T., Kohno, S., et al. (2002). Multidrug efflux systems play an important role in the invasiveness of Pseudomonas aeruginosa. J. Exp. Med. 196, 109-118. doi: 10.1084/jem. 20020005

Hogan, D. A., Vik, A., and Kolter, R. (2004). A Pseudomonas aeruginosa quorumsensing molecule influences Candida albicans morphology. Mol. Microbiol. 54, 1212-1223. doi: 10.1111/j.1365-2958.2004.04349.x

Houry, A., Gohar, M., Deschamps, J., Tischenko, E., Aymerich, S., Gruss, A., et al. (2012). Bacterial swimmers that infiltrate and take over the biofilm matrix. Proc. Natl. Acad. Sci. U.S.A. 109, 13088-13093. doi: 10.1073/pnas. 1200 791109

Jang, S. (2016). Multidrug efflux pumps in Staphylococcus aureus and their clinical implications. J. Microbiol. 54, 1-8. doi: 10.1007/s12275-016-5159-Z

Jin, Y., Yang, H., Qiao, M., and Jin, S. (2011). MexT regulates the type III secretion system through MexS and PtrC in Pseudomonas aeruginosa. J. Bacteriol. 193, 399-410. doi: 10.1128/JB.01079-10

Kawamura-Sato, K., Shibayama, K., Horii, T., Iimuma, Y., Arakawa, Y., and Ohta, M. (1999). Role of multiple efflux pumps in Escherichia coli in indole expulsion. FEMS Microbiol. Lett. 179, 345-352. doi: 10.1111/j.15746968.1999.tb08748.x

Kaye, K. S., and Pogue, J. M. (2015). Infections caused by resistant gram-negative bacteria: epidemiology and management. Pharmacotherapy 35, 949-962. doi: 10.1002/phar.1636

Khoramian-Falsafi, T., Harayama, S., Kutsukake, K., and Pechere, J. C. (1990). Effect of motility and chemotaxis on the invasion of Salmonella typhimurium into HeLa cells. Microb. Pathog. 9, 47-53. doi: 10.1016/0882-4010(90) 90039-S

Kim, I. Y., Pusey, P. L., Zhao, Y., Korban, S. S., Choi, H., and Kim, K. K. (2012). Controlled release of Pantoea agglomerans E325 for biocontrol of fire blight disease of apple. J. Control. Release 161, 109-115. doi: 10.1016/j.jconrel.2012.03.028

Köhler, T., Delden, C. V., Van Delden, C., Curty, L. K., Hamzehpour, M. M., and Pechere, J. C. (2001). Overexpression of the MexEF-OprN multidrug efflux system affects cell-to-cell signaling in Pseudomonas aeruginosa. J. Bacteriol. 183, 5213-5222. doi: 10.1128/JB.183.18.5213 
Lamarche, M. G., and Deziel, E. (2011). MexEF-OprN Efflux Pump Exports the Pseudomonas Quinolone Signal (PQS) Precursor HHQ (4-hydroxy2-heptylquinoline). PLoS ONE 6:e24310. doi: 10.1371/journal.pone. 0024310

Lee, J., Wu, J., Deng, Y., Wang, J., Wang, C., Wang, J., et al. (2013). A cellcell communication signal integrates quorum sensing and stress response. Nat. Chem. Biol. 9, 339-343. doi: 10.1038/nchembio.1225

Lee, J., and Zhang, L. (2015). The hierarchy quorum sensing network in Pseudomonas aeruginosa. Protein Cell 6, 26-41. doi: 10.1007/s13238-0140100-x

Lee, J. H., Wood, T. K., and Lee, J. (2015). Roles of indole as an interspecies and interkingdom signaling molecule. Trends Microbiol. 23, 707-718. doi: 10.1016/j.tim.2015.08.001

Lee, J. S., Ryu, H. R., Cha, J. Y., and Baik, H. S. (2015). The hrp pathogenicity island of Pseudomonas syringae pv. tomato DC3000 is induced by plant phenolic acids. J. Microbiol. 53, 725-731. doi: 10.1007/s12275-015-5256-4

Li, X. Z., Nikaido, H., and Poole, K. (1995). Role of mexA-mexB-oprM in antibiotic efflux in Pseudomonas aeruginosa. Antimicrob. Agents Chemother. 39, 19481953. doi: 10.1128/AAC.39.9.1948

Li, X.-Z., Plésiat, P., and Nikaido, H. (2015). The challenge of efflux-mediated antibiotic resistance in Gram-negative bacteria. Clin. Microbiol. Rev. 28, 337418.

Liao, J., Schurr, M. J., and Sauer, K. (2013). The MerR-like regulator BrlR confers biofilm tolerance by activating multidrug efflux pumps in Pseudomonas aeruginosa biofilms. J. Bacteriol. 195, 3352-3363. doi: 10.1128/JB. 00318-13

Lin, Y. T., Huang, Y. W., Chen, S. J., Chang, C. W., and Yang, T. C. (2015). The SmeYZ efflux pump of Stenotrophomonas maltophilia contributes to drug resistance, virulence-related characteristics, and virulence in mice. Antimicrob. Agents Chemother. 59, 4067-4073. doi: 10.1128/aac.00372-15

Linares, J. F., Lopez, J. A., Camafeita, E., Albar, J. P., Rojo, F., and Martinez, J. L. (2005). Overexpression of the multidrug efflux pumps MexCD-OprJ and MexEF-OprN is associated with a reduction of type III secretion in Pseudomonas aeruginosa. J. Bacteriol. 187, 1384-1391. doi: 10.1128/JB.187.4.1384-1391.2005

Lindemann, A., Koch, M., Pessi, G., Muller, A. J., Balsiger, S., Hennecke, H., et al. (2010). Host-specific symbiotic requirement of BdeAB, a RegR-controlled RND-type efflux system in Bradyrhizobium japonicum. FEMS Microbiol. Lett. 312, 184-191. doi: 10.1111/j.1574-6968.2010.02115.x

Luong, T. T., Newell, S. W., and Lee, C. Y. (2003). Mgr, a novel global regulator in Staphylococcus aureus. J. Bacteriol. 185, 3703-3710. doi: 10.1128/JB.185.13.3703-3710.2003

Maggiorani Valecillos, A., Rodriguez Palenzuela, P., and Lopez-Solanilla, E. (2006). The role of several multidrug resistance systems in Erwinia chrysanthemi pathogenesis. Mol. Plant Microbe Interact. 19, 607-613. doi: 10.1094/MPMI19-0607

Martinez, J. L. (2014). Interkingdom signaling and its consequences for human health. Virulence 5, 243-244. doi: 10.4161/viru.28073

Martinez, J. L., and Baquero, F. (2002). Interactions among strategies associated with bacterial infection: pathogenicity, epidemicity, and antibiotic resistance. Clin. Microbiol. Rev. 15, 647-679. doi: 10.1128/CMR.15.4.647679.2002

Martinez, J. L., Sanchez, M. B., Martinez-Solano, L., Hernandez, A., Garmendia, L., Fajardo, A., et al. (2009). Functional role of bacterial multidrug efflux pumps in microbial natural ecosystems. FEMS Microbiol. Rev. 33, 430-449. doi: 10.1111/j.1574-6976.2008.00157.x

Martinez-Solano, L., Macia, M. D., Fajardo, A., Oliver, A., and Martinez, J. L. (2008). Chronic Pseudomonas aeruginosa infection in chronic obstructive pulmonary disease. Clin. Infect. Dis. 47, 1526-1533. doi: 10.1086/ 593186

Maseda, H., Sawada, I., Saito, K., Uchiyama, H., Nakae, T., and Nomura, N. (2004). Enhancement of the mexAB-oprM efflux pump expression by a quorumsensing autoinducer and its cancellation by a regulator, MexT, of the mexEFoprN efflux pump operon in Pseudomonas aeruginosa. Antimicrob. Agents Chemother. 48, 1320-1328. doi: 10.1128/AAC.48.4.1320-1328.2004

Méar, J.-B., Kipnis, E., Faure, E., Dessein, R., Schurtz, G., Faure, K., et al. (2013). Candida albicans and Pseudomonas aeruginosa interactions: more than an opportunistic criminal association? Méd. Mal. Infect. 43, 146-151. doi: 10.1016/j.medmal.2013.02.005

Middleton, B., Rodgers, H. H. C., Cámara, M., Knox, A. J., Williams, P., and Hardman, A. (2002). Direct detection of $\mathrm{N}$-acylhomoserine lactones in cystic fibrosis sputum. FEMS Microbiol. Lett. 207, 1-7. doi: 10.1016/S03781097(01)00556-0

Mima, T., and Schweizer, H. P. (2010). The BpeAB-OprB efflux pump of Burkholderia pseudomallei $1026 \mathrm{~b}$ does not play a role in quorum sensing, virulence factor production, or extrusion of aminoglycosides but is a broadspectrum drug efflux system. Antimicrob. Agents Chemother. 54, 3113-3120. doi: 10.1128/AAC.01803-09

Minagawa, S., Inami, H., Kato, T., Sawada, S., Yasuki, T., Miyairi, S., et al. (2012). RND type efflux pump system MexAB-OprM of Pseudomonas aeruginosa selects bacterial languages, 3-oxo-acyl-homoserine lactones, for cell-to-cell communication. BMC Microbiol. 12:70. doi: 10.1186/14712180-12-70

Neyrolles, O., and Guilhot, C. (2011). Recent advances in deciphering the contribution of Mycobacterium tuberculosis lipids to pathogenesis. Tuberculosis 91, 187-195. doi: 10.1016/j.tube.2011.01.002

Nikaido, E., Giraud, E., Baucheron, S., Yamasaki, S., Wiedemann, A., Okamoto, K., et al. (2012). Effects of indole on drug resistance and virulence of Salmonella enterica serovar typhimurium revealed by genome-wide analyses. Gut Pathog. 4:5. doi: 10.1186/1757-4749-4-5

Nikaido, E., Shirosaka, I., Yamaguchi, A., and Nishino, K. (2011). Regulation of the AcrAB multidrug efflux pump in Salmonella enterica serovar typhimurium in response to indole and paraquat. Microbiology 157(Pt. 3), 648-655. doi: 10.1099/mic.0.045757-0

Nikaido, E., Yamaguchi, A., and Nishino, K. (2008). AcrAB multidrug efflux pump regulation in Salmonella enterica serovar typhimurium by RamA in response to environmental signals. J. Biol. Chem. 283, 24245-24253. doi: 10.1074/jbc.M804544200

Olivares, J., Alvarez-Ortega, C., Linares, J. F., Rojo, F., Kohler, T., and Martinez, J. L. (2012). Overproduction of the multidrug efflux pump MexEF-OprN does not impair Pseudomonas aeruginosa fitness in competition tests, but produces specific changes in bacterial regulatory networks. Environ. Microbiol. 14, 19681981. doi: 10.1111/j.1462-2920.2012.02727.x

Oliver, A., Mulet, X., Lopez-Causape, C., and Juan, C. (2015). The increasing threat of Pseudomonas aeruginosa high-risk clones. Drug Resist. Updat. 2, 41-59. doi: 10.1016/j.drup.2015.08.002

Palumbo, J. D., Kado, C. I., and Phillips, D. A. (1998). An isoflavonoidinducible efflux pump in Agrobacterium tumefaciens is involved in competitive colonization of roots. J. Bacteriol. 180, 3107-3113.

Papadopoulos, C. J., Carson, C. F., Chang, B. J., and Riley, T. V. (2008). Role of the MexAB-OprM efflux pump of Pseudomonas aeruginosa in tolerance to tea tree (Melaleuca alternifolia) oil and its monoterpene components terpinen-4-ol, 1,8-cineole, and alpha-terpineol. Appl. Environ. Microbiol. 74, 1932-1935. doi: 10.1128/AEM.02334-07

Pearson, J. P., Van Delden, C., and Iglewski, B. H. (1999). Active efflux and diffusion are involved in transport of Pseudomonas aeruginosa cell-to-cell signals. J. Bacteriol. 181, 1203-1210.

Piddock, L. J. (2006a). Clinically relevant chromosomally encoded multidrug resistance efflux pumps in bacteria. Clin. Microbiol. Rev. 19, 382-402. doi: 10.1128/CMR.19.2.382-402.2006

Piddock, L. J. (2006b). Multidrug-resistance efflux pumps - not just for resistance. Nat. Rev. Microbiol. 4, 629-636. doi: 10.1038/nrmicro1464

Pinero-Fernandez, S., Chimerel, C., Keyser, U. F., and Summers, D. K. (2011). Indole transport across Escherichia coli membranes. J. Bacteriol. 193, 17931798. doi: 10.1128/JB.01477-10

Pletzer, D., and Weingart, H. (2014). Characterization and regulation of the resistance-nodulation-cell division-type multidrug efflux pumps MdtABC and MdtUVW from the fire blight pathogen Erwinia amylovora. BMC Microbiol. 14:185. doi: 10.1186/1471-2180-14-185

Poole, K. (2001). Multidrug resistance in Gram-negative bacteria. Curr. Opin. Microbiol. 4, 500-508. doi: 10.1016/S1369-5274(00)00242-3

Prouty, A. M., Brodsky, I. E., Falkow, S., and Gunn, J. S. (2004). Bile-salt-mediated induction of antimicrobial and bile resistance in Salmonella typhimurium. Microbiology 150(Pt. 4), 775-783. doi: 10.1099/mic.0.26769-0 
Pusey, P. L., Stockwell, V. O., Reardon, C. L., Smits, T. H., and Duffy, B. (2011). Antibiosis activity of Pantoea agglomerans biocontrol strain E325 against Erwinia amylovora on apple flower stigmas. Phytopathology 101, 1234-1241. doi: 10.1094/phyto-09-10-0253

Quillin, S. J., Schwartz, K. T., and Leber, J. H. (2011). The novel Listeria monocytogenes bile sensor BrtA controls expression of the cholic acid efflux pump MdrT. Mol. Microbiol. 81, 129-142. doi: 10.1111/j.13652958.2011.07683.x

Randall, L. P., and Woodward, M. J. (2002). The multiple antibiotic resistance (mar) locus and its significance. Res. Vet. Sci. 72, 87-93. doi: 10.1053/rvsc.2001.0537

Ravirala, R. S., Barabote, R. D., Wheeler, D. M., Reverchon, S., Tatum, O., Malouf, J., et al. (2007). Efflux pump gene expression in Erwinia chrysanthemi is induced by exposure to phenolic acids. Mol. Plant Microbe Interact. 20, 313-320. doi: 10.1094/MPMI-20-3-0313

Redgrave, L. S., Sutton, S. B., Webber, M. A., and Piddock, L. J. (2014). Fluoroquinolone resistance: mechanisms, impact on bacteria, and role in evolutionary success. Trends Microbiol. 22, 438-445. doi: 10.1016/j.tim.2014.04.007

Rosenberg, E. Y., Bertenthal, D., Nilles, M. L., Bertrand, K. P., and Nikaido, H. (2003). Bile salts and fatty acids induce the expression of Escherichia coli AcrAB multidrug efflux pump through their interaction with Rob regulatory protein. Mol. Microbiol. 48, 1609-1619. doi: 10.1046/j.1365-2958.2003. 03531.x

Rossbach, S., Kunze, K., Albert, S., Zehner, S., and Gottfert, M. (2014). The Sinorhizobium meliloti EmrAB efflux system is regulated by flavonoids through a TetR-like regulator (EmrR). Mol. Plant Microbe Interact. 27, 379-387. doi: 10.1094/MPMI-09-13-0282-R

Sakhtah, H., Koyama, L., Zhang, Y., Morales, D. K., Fields, B. L., PriceWhelan, A., et al. (2016). The Pseudomonas aeruginosa efflux pump MexGHI-OpmD transports a natural phenazine that controls gene expression and biofilm development. Proc. Natl. Acad. Sci. U.S.A. 113:E3538-47. doi: 10.1073/pnas.1600424113

Sanchez, P., Alonso, A., and Martinez, J. L. (2004). Regulatory regions of smeDEF in Stenotrophomonas maltophilia strains expressing different amounts of the multidrug efflux pump SmeDEF. Antimicrob. Agents Chemother. 48, 2274 2276. doi: 10.1128/AAC.48.6.2274-2276.2004

Sanchez, P., Linares, J. F., Ruiz-Diez, B., Campanario, E., Navas, A., Baquero, F., et al. (2002). Fitness of in vitro selected Pseudomonas aeruginosa nalB and nfxB multidrug resistant mutants. J. Antimicrob. Chemother. 50, 657-664. doi: 10.1093/jac/dkf185

Santos, M. R., Marques, A. T., Becker, J. D., and Moreira, L. M. (2014). The Sinorhizobium meliloti EmrR regulator is required for efficient colonization of Medicago sativa root nodules. Mol. Plant Microbe Interact. 27, 388-399. doi: 10.1094/MPMI-09-13-0284-R

Sawada, I., Maseda, H., Nakae, T., Uchiyama, H., and Nomura, N. (2004). A quorum-sensing autoinducer enhances the mexAB-oprM effluxpump expression without the MexR-mediated regulation in Pseudomonas aeruginosa. Microbiol. Immunol. 48, 435-439. doi: 10.1111/j.1348-0421.2004. tb03533. X

Shimada, Y., Kinoshita, M., Harada, K., Mizutani, M., Masahata, K., Kayama, H., et al. (2013). Commensal bacteria-dependent indole production enhances epithelial barrier function in the colon. PLOS ONE 8:e80604. doi: 10.1371/journal.pone.0080604

Smith, H. E., and Blair, J. M. (2014). Redundancy in the periplasmic adaptor proteins AcrA and AcrE provides resilience and an ability to export substrates of multidrug efflux. J. Antimicrob. Chemother. 69, 982-987. doi: 10.1093/jac/dkt481

Smith, R. S., Fedyk, E. R., Springer, T. A., Mukaida, N., Iglewski, B. H., and Phipps, R. P. (2001). IL-8 production in human lung fibroblasts and epithelial cells activated by the Pseudomonas autoinducer N-3-oxododecanoyl homoserine lactone is transcriptionally regulated by NF-kappa B and activator protein-2. J. Immunol. 167, 366-374. doi: 10.4049/jimmunol.167.1.366

Soto, S. M. (2013). Role of efflux pumps in the antibiotic resistance of bacteria embedded in a biofilm. Virulence 4, 223-229. doi: 10.4161/viru.23724

Spaniol, V., Bernhard, S., and Aebi, C. (2015). Moraxella catarrhalis AcrABOprM efflux pump contributes to antimicrobial resistance and is enhanced during cold shock response. Antimicrob. Agents Chemother. 59, 1886-1894. doi: 10.1128/AAC.03727-14

Spaniol, V., Wyder, S., and Aebi, C. (2013). RNA-Seq-based analysis of the physiologic cold shock-induced changes in Moraxella catarrhalis gene expression. PLoS ONE 8:e68298. doi: 10.1371/journal.pone.0068298

Swift, S., and Downie, J. A. (2001). Quorum sensing as a population-densitydependent determinant of bacterial physiology. Adv. Microbial Physiol. 45, 199-270.

Szekely, R., and Cole, S. T. (2016). Mechanistic insight into mycobacterial MmpL protein function. Mol. Microbiol. 99, 831-834. doi: 10.1111/mmi.13306

Takeshima, K., Hidaka, T., Wei, M., Yokoyama, T., Minamisawa, K., Mitsui, H., et al. (2013). Involvement of a novel genistein-inducible multidrug efflux pump of Bradyrhizobium japonicum early in the interaction with Glycine max (L.) Merr. Microbes Environ. 28, 414-421. doi: 10.1264/jsme2.ME13057

Talwalkar, J. S., and Murray, T. S. (2016). The approach to Pseudomonas aeruginosa in cystic fibrosis. Clin. Chest Med. 37, 69-81. doi: 10.1016/j.ccm.2015.10.004

Tateda, K., Ishii, Y., Horikawa, M., Matsumoto, T., Miyairi, S., Pechere, J. C., et al. (2003). The Pseudomonas aeruginosa autoinducer N-3-oxododecanoyl homoserine lactone accelerates apoptosis in macrophages and neutrophils. Infect. Immun. 71, 5785-5793. doi: 10.1128/IAI.71.10.5785-5793.2003

Taylor, D. L., Bina, X. R., and Bina, J. E. (2012). Vibrio cholerae VexH encodes a multiple drug efflux pump that contributes to the production of cholera toxin and the toxin co-regulated pilus. PLOS ONE 7:e38208. doi: 10.1371/journal.pone.0038208

Thanassi, D. G., Cheng, L. W., and Nikaido, H. (1997). Active efflux of bile salts by Escherichia coli. J. Bacteriol. 179, 2512-2518.

Ulrich, R. L., Deshazer, D., Brueggemann, E. E., Hines, H. B., Oyston, P. C., and Jeddeloh, J. A. (2004). Role of quorum sensing in the pathogenicity of Burkholderia pseudomallei. J. Med. Microbiol. 53(Pt. 11), 1053-1064. doi: 10.1099/jmm.0.45661-0

Vargas, P., Farias, G. A., Nogales, J., Prada, H., Carvajal, V., Baron, M., et al. (2013). Plant flavonoids target Pseudomonas syringae pv. tomato DC3000 flagella and type III secretion system. Environ. Microbiol. Rep. 5, 841-850. doi: $10.1111 / 1758-2229.12086$

Vargas, P., Felipe, A., Michan, C., and Gallegos, M. T. (2011). Induction of Pseudomonas syringae pv. tomato DC3000 MexAB-OprM multidrug efflux pump by flavonoids is mediated by the repressor PmeR. Mol. Plant Microbe Interact. 24, 1207-1219. doi: 10.1094/MPMI-03-11-0077

Vila, J., and Martínez, J. L. (2008). Clinical impact of the over-expression of efflux pump in nonfermentative Gram-negative bacilli, development of efflux pump inhibitors. Curr. Drug Targets 9, 797-807. doi: 10.2174/138945008785747806

Wagner, C., Zimmermann, S., Brenner-Weiss, G., Hug, F., Prior, B., Obst, U., et al. (2007). The quorum-sensing molecule N-3-oxododecanoyl homoserine lactone (3OC12-HSL) enhances the host defence by activating human polymorphonuclear neutrophils (PMN). Anal. Bioanal. Chem. 387, 481-487. doi: 10.1007/s00216-006-0698-5

Wagner, V. E., and Iglewski, B. H. (2008). Pseudomonas aeruginosa biofilms in CF infection. Clin. Rev. Allergy Immunol. 35, 124-134. doi: 10.1007/s12016-0088079-9

Webber, M. A., Bailey, A. M., Blair, J. M., Morgan, E., Stevens, M. P., Hinton, J. C., et al. (2009). The global consequence of disruption of the AcrAB-TolC efflux pump in Salmonella enterica includes reduced expression of SPI-1 and other attributes required to infect the host. J. Bacteriol. 191, 4276-4285. doi: 10.1128/JB.00363-09

Williams, P. (2007). Quorum sensing, communication and cross-kingdom signalling in the bacterial world. Microbiology 153(Pt. 12), 3923-3938. doi: 10.1099/mic.0.2007/012856-0

Williams, P., Winzer, K., Chan, W. C., and Camara, M. (2007). Look who's talking: communication and quorum sensing in the bacterial world. Philos. Trans. $R$. Soc. Lond. B Biol. Sci. 362, 1119-1134. doi: 10.1098/rstb.2007.2039

Wongtrakoongate, P., Tumapa, S., and Tungpradabkul, S. (2012). Regulation of a quorum sensing system by stationary phase sigma factor RpoS and their coregulation of target genes in Burkholderia pseudomallei. Microbiol. Immunol. 56, 281-294. doi: 10.1111/j.1348-0421.2012.00447.x

Yamaguchi, A., Nakashima, R., and Sakurai, K. (2015). Structural basis of RND-type multidrug exporters. Front. Microbiol. 6:327. doi: 10.3389/fmicb.2015.00327 
Zhang, L., Li, X. Z., and Poole, K. (2001). SmeDEF multidrug efflux pump contributes to intrinsic multidrug resistance in Stenotrophomonas maltophilia. Antimicrob. Agents Chemother. 45, 3497-3503. doi: 10.1128/AAC.45.12.34973503.2001

Zhang, L., and Mah, T. F. (2008). Involvement of a novel efflux system in biofilm-specific resistance to antibiotics. J. Bacteriol. 190, 4447-4452. doi: 10.1128/JB.01655-07

Zimmermann, S., Wagner, C., Müller, W., Brenner-Weiss, G., Hug, F., Prior, B., et al. (2006). Induction of neutrophil chemotaxis by the quorum-sensing molecule N-(3-oxododecanoyl)-L-homoserine lactone. Infect. Immun. 74, 5687-5692. doi: 10.1128/IAI.01940-05
Conflict of Interest Statement: The authors declare that the research was conducted in the absence of any commercial or financial relationships that could be construed as a potential conflict of interest.

Copyright (c) 2016 Alcalde-Rico, Hernando-Amado, Blanco and Martínez. This is an open-access article distributed under the terms of the Creative Commons Attribution License (CC BY). The use, distribution or reproduction in other forums is permitted, provided the original author(s) or licensor are credited and that the original publication in this journal is cited, in accordance with accepted academic practice. No use, distribution or reproduction is permitted which does not comply with these terms. 